\title{
Stable structure and magnetic state of ultrathin CrAs films on GaAs(001): A density functional theory study
}

\author{
S. Javad Hashemifar, ${ }^{1,2}$ Peter Kratzer, ${ }^{2}$ and Matthias Scheffler ${ }^{3}$ \\ ${ }^{1}$ Department of Physics and Nanotechnology and Advanced Materials Institute, Isfahan University of Technology, \\ 84156-83111 Isfahan, Iran \\ ${ }^{2}$ Fakultät für Physik, Universität Duisburg-Essen, D-47048 Duisburg, Germany \\ ${ }^{3}$ Fritz-Haber-Institut der Max-Planck-Gesellschaft, D-14195 Berlin, Germany
}

(Received 3 September 2010; revised manuscript received 30 November 2010; published 14 December 2010)

\begin{abstract}
Density functional theory calculations using the pseudopotential-plane-wave approach are employed to investigate the structural and magnetic properties of epitaxial $\mathrm{CrAs}$ thin films on $\mathrm{GaAs}(001)$. Motivated by recent reports of ferromagnetism in this system, we compare zinc-blende CrAs films (continuing the lattice structure of the GaAs substrate) and CrAs films with a bulklike orthorhombic structure epitaxially matched to three units of the $\mathrm{GaAs}(001)$ lattice. We find that even for very thin films with three Cr layers the bulklike crystal structure is energetically more favorable than zinc-blende CrAs on GaAs(001). CrAs films with orthorhombic structure, even if under epitaxial strain, preserve the antiferromagnetic order of CrAs bulk. In the light of our calculations, it appears likely that the magnetic hysteresis loop measured in ultrathin $\mathrm{CrAs} / \mathrm{GaAs}(001)$ films originates from uncompensated antiferromagnetic moments near the $\mathrm{CrAs} / \mathrm{GaAs}$ interface. In conclusion, our results do not support earlier proposals that thick CrAs films could be employed as perfectly matched spin-injection electrode on GaAs.
\end{abstract}

DOI: 10.1103/PhysRevB.82.214417

PACS number(s): 75.70.Ak, 75.25.-j, 68.55.-a

\section{MOTIVATION AND BACKGROUND}

Spin injection from a ferromagnet into a semiconductor is a major prerequisite for semiconductor spintronics. In recent years it has become clear that the efficiency of spin injection not only depends on the degree of spin polarization in the ferromagnet but the ferromagnet-semiconductor interface also plays a crucial role. Using electrodes fabricated from conventional ferromagnetic (FM) materials, such as $\mathrm{Fe}$ or $\mathrm{Co}$, only a limited amount of spin-polarized current could be injected into GaAs. ${ }^{1,2}$ As possible reasons, the conductivity mismatch in the metal and the (doped) semiconductor has been discussed ${ }^{3}$ but the unsatisfactory results could also be due to technological problems in fabricating high-quality interfaces.

As an alternative, Akinaga et al. ${ }^{4}$ suggested to grow CrAs epitaxially, in the zinc-blende (ZB) structure, on GaAs. As advantages over conventional ferromagnetic electrodes, it has been pointed out that the use of a zinc-blende CrAs layer on GaAs not only avoids (possibly detrimental) interface states ${ }^{5}$ but in addition zinc-blende CrAs is a half-metallic ferromagnet according to DFT calculations, i.e., it offers the highest possible spin polarization of $100 \%$. By growing a superlattice of alternating CrAs and GaAs layers, Akinaga et al. managed to fabricate heterostructures showing ferromagnetic behavior at room temperature. Following this work, several research groups studied epitaxial CrAs films on GaAs, ${ }^{6-11}$ and confirmed the ferromagnetic hysteresis of the samples. The saturation magnetization was found to correspond to about $2 \mu_{B}$ up to $3 \mu_{B}$ per $\mathrm{Cr}$ atom. However, the remanent magnetization was typically much lower, less than $10 \%$ of the saturation magnetization. Transmission electron microscopy (TEM) images of the samples demonstrated pseudomorphic growth of CrAs on GaAs, and most researchers claimed the formation of zinc-blende CrAs. However,
Etgens et al. ${ }^{12}$ using in situ reflection-high-energy electron diffraction and grazing-incidence x-ray diffraction (GIXD) could not find any evidence of the $\mathrm{ZB}$ CrAs structure even for a $12 \AA$ thin layer. They attributed the measured roomtemperature ferromagnetic signal in this system to a $\mathrm{CrAs}$ phase with orthorhombic structure but different from bulk CrAs.

As bulk material, CrAs forms orthorhombic crystals with the MnP structure that can be envisaged as a (simpler) NiAstype crystal structure in which $\mathrm{Cr}$ atoms are alternately displaced in opposite directions. In this phase, CrAs shows antiferromagnetic $(\mathrm{AF})$ behavior, and its spin structure has been described as a double helix. ${ }^{13}$ Hence, bulk CrAs is not very attractive for spintronics. The use of CrAs for spin injection relies crucially on the modification of its magnetic and/or structural properties in epitaxial films. However, from the theoretical point of view, first-principles studies indicate that ZB binary half metals are rarely stabilized by coherent epitaxy. Xie et al. ${ }^{14}$ compared density functional total energies of the bulk $3 d$ transition-metal chalcogenides in $\mathrm{ZB}$ and NiAs-type structures and found a rather high energy for the metastable ZB CrAs. Zhao et al. ${ }^{15}$ performed a more accurate comparison, taking into account the tetragonal distortion in the films induced by the substrate. They compared the total energies of the tetragonally distorted bulk $\mathrm{ZB}$ and the NiAs structure of six binary alloys (including CrAs) and looked for the critical lattice parameter after which the tetragonally distorted $\mathrm{ZB}$ structure becomes more stable. They concluded that there is no such transition in CrAs, and $\mathrm{CrSe}$ is the only alloy for which the transition to a ferromagnetic ZB structure is thermodynamically favorable for an epitaxially strained film. These findings cast some doubt on the claimed ZB structure of CrAs films. Moreover, the magnetic moment in half-metallic ZB CrAs is calculated to be $3 \mu_{B}$ (Ref. 16) while the values derived from the experimental saturation magnetization are markedly lower. 
In the present work, we carry out a theoretical study of CrAs both as distorted bulk material and as thin film on GaAs in order to explore different explanations for the observed ferromagnetic behavior. Epitaxially strained $\mathrm{CrAs}$ may possibly retain the $\mathrm{MnP}$ or NiAs crystal structure but may change its spin structure to ferromagnetic ordering. Alternatively, both the structural and magnetic order of bulk CrAs might be retained in the films, but the ferromagnetic signal arises due to uncompensated magnetic moments at the interface between GaAs and CrAs.

\section{METHOD OF CALCULATION}

Our density functional theory (DFT) calculations were performed by using the plane-wave pseudopotential method implemented in the QUANTUM-ESPRESSO package. ${ }^{17,18}$ In this method the wave functions of valence electrons are expanded into plane waves while the core electrons are treated with the ultrasoft pseudopotential technique. ${ }^{19}$ The valence electron shells in $\mathrm{Cr}, \mathrm{Ga}$, and As pseudopotentials are $3 s^{2} 3 p^{6} 4 s^{1} 4 p^{0} 3 d^{5}, 3 d^{10} 4 s^{2} 4 p^{1}$, and $4 s^{2} 4 p^{3}$, respectively. The performance of the pseudopotentials was tested by comparing the results for the bulk structures to all-electron calculations (linearized augmented plane wave method ${ }^{20}$ ). The generalized gradient approximation (GGA) to the exchangecorrelation potential in form of the Perdew, Burke, and Ernzerhof (PBE) functional was used in this work. ${ }^{21}$ After convergence tests, an energy cutoff of 30 Ry for the planewave expansion and 350 Ry for the Fourier expansion of electron density were selected. The Brillouin zone integrations were performed by using the Marzari-Vanderbilt coldsmearing method, a broadening parameter of $0.02 \mathrm{Ry}$, and k-point meshes of $4 \times 12 \times 1$ and $6 \times 12 \times 1$ points for MnPtype $\mathrm{CrAs} / \mathrm{GaAs}$ and $2 \times 1$ reconstructed $\mathrm{ZB} \mathrm{CrAs} / \mathrm{GaAs}$ interfaces, respectively. It was verified that these $\mathbf{k}$-point meshes yield total energies with an error of less than 0.1 mRy. All the structures have been optimized to achieve the minimum energy by accurate relaxation of the atomic positions up to the energy accuracy of $0.1 \mathrm{mRy}$ and forces of less than $1 \mathrm{mRy} / \mathrm{bohr}$.

\section{BULK CrAs}

The stable (ground state) structure of bulk CrAs is an MnP-type orthorhombic lattice (space group Pnma) with a double-spiral antiferromagnetic spin structure along the c axis. ${ }^{13}$ In this structure, both $\mathrm{Mn}$ and As atoms are sitting on the 4c Wyckoff positions: $\left(x, \frac{1}{4}, z\right),\left(-x, \frac{3}{4},-z\right),\left(\frac{1}{2}-x, \frac{3}{4}, \frac{1}{2}\right.$ $+z),\left(\frac{1}{2}+x, \frac{1}{4}, \frac{1}{2}-z\right)$. It is evident from Fig. 1 that the MnPtype structure is a slightly distorted NiAs-type structure. Since the distortions in thin films and in bulk could be different, both structures were included in our search for the stable state of the CrAs alloy. In the MnP-type lattice (MP), compared to NiAs-type (NA), the $\mathrm{Cr} 2$ and $\mathrm{Cr} 3$ atoms move toward each other, leading to substantial change in the magnetic state of the system (will be shown below).

In the MP ground state, CrAs has a noncollinear spin structure in which all $\mathrm{Cr}$ magnetic moments are in the $x y$ plane and the angles between them are: $\langle\mathrm{Cr} 1, \mathrm{Cr} 2\rangle$

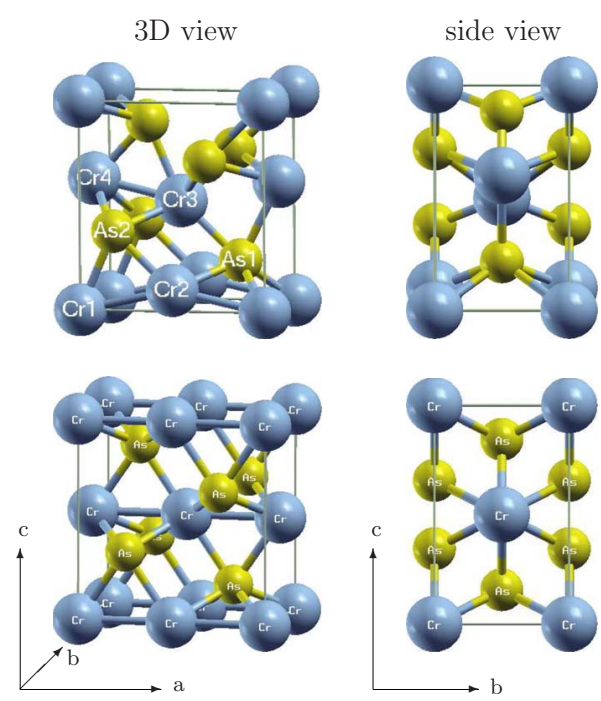

FIG. 1. (Color online) Three-dimensional and side views of the orthorhombic MnP-type (top panel) and NiAs-type (bottom panel) crystal structures. Note that the Wyckoff positions are shifted to put a $\mathrm{Cr}$ atom at the origin.

$=\langle\mathrm{Cr} 3, \mathrm{Cr} 4\rangle=-120^{\circ} \quad$ and $\langle\mathrm{Cr} 1, \mathrm{Cr} 4\rangle=\langle\mathrm{Cr} 2, \mathrm{Cr} 3\rangle=183^{\circ} . .^{13}$ Thus, CrAs shows antiferromagnetic behavior. In this work, we restrict ourselves to collinear magnetic structures, as the energy differences due to noncollinear structures are expected to be small. Approximating all angles by $180^{\circ}$ leads to the collinear structure closest to the real ground state of CrAs. In Fig. 2 this structure, called AFh, is shown along with other possible collinear antiferromagnetic states for both the MP and NA crystal structure. In addition to these antiferromagnetic states we have also studied the FM state of MP and NA structures, as well as the AF and FM states of the $\mathrm{ZB}$ lattice. In the following, two sets of calculations are performed for all these states: calculations with full structural optimization, and calculations where epitaxial constraints are applied.

In the fully optimized calculations all lattice parameters and internal atomic positions were relaxed to find out the theoretical ground state of the system. By fitting the BirchMurnaghan equation of state to the obtained total energies as a function of volume, structural properties of the systems were determined. The results are listed in Table I. The corresponding energy-volume curves are also shown in Fig. 3. It is observed from Table I that MP-AFh is the most stable among the states considered. This is in agreement with the experimental ground state. Theoretical lattice constants in
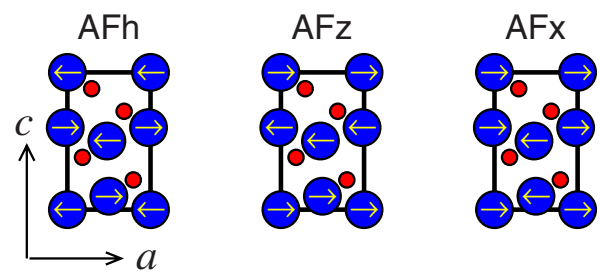

FIG. 2. (Color online) Schematic representation of three selected AF states for MP-CrAs. Big circles indicate $\mathrm{Cr}$ atoms and lattice vectors are shown in the lower left corner. 
TABLE I. The calculated structural properties of the different lattice types and magnetic states of CrAs; $V_{0}\left(\AA^{3}\right)$ : volume per CrAs pair, $a, b$, and $c(\AA)$ : lattice constants, $x$ and $z$ : internal parameters in relative coordinates, $B$ (kbar): bulk modulus, $E_{\text {coh }}(\mathrm{eV})$ : cohesive energy per CrAs pair. The values with an asterisk are obtained in the lattice-matched structures. The experimental data are measured at $80 \mathrm{~K}$ (Ref. 13 ).

\begin{tabular}{|c|c|c|c|c|c|c|c|c|c|c|c|}
\hline & & $V_{0}$ & $a$ & $b$ & $c$ & $c^{*}$ & $(x, z)_{\mathrm{Cr}}$ & $(x, z)_{\mathrm{As}}$ & B & $E_{\mathrm{coh}}$ & $E_{\mathrm{coh}}^{*}$ \\
\hline \multirow[t]{3}{*}{ MP } & $\mathrm{AFh}$ & 31.50 & 5.58 & 3.66 & 6.17 & & $0.012,0.201$ & $0.195,0.582$ & 869 & 7.250 & \\
\hline & $\mathrm{AFz}$ & 31.67 & 5.73 & 3.61 & 6.13 & & $0.007,0.206$ & $0.210,0.586$ & 760 & 7.183 & \\
\hline & FM & 32.24 & 5.70 & 3.60 & 6.28 & & $0.002,0.217$ & $0.216,0.581$ & 835 & 7.215 & \\
\hline \multirow[t]{4}{*}{ NA } & $\mathrm{AFh}$ & 33.00 & 5.79 & 3.74 & 6.09 & 5.69 & $0,0.25$ & $0.25,0.583$ & 942 & 7.177 & 7.024 \\
\hline & $\mathrm{AFx}$ & 33.00 & 5.31 & 3.79 & 6.57 & 6.04 & $0,0.25$ & $0.25,0.583$ & 987 & 7.230 & 6.890 \\
\hline & $\mathrm{AFz}$ & 32.61 & 5.92 & 3.66 & 6.03 & 5.72 & $0,0.25$ & $0.25,0.583$ & 877 & 7.158 & 6.981 \\
\hline & FM & 33.01 & 5.69 & 3.66 & 6.34 & 5.94 & $0,0.25$ & $0.25,0.583$ & 860 & 7.193 & 6.974 \\
\hline \multirow[t]{2}{*}{ ZB } & $\mathrm{AF}$ & 44.20 & 5.61 & & & 5.28 & & & 605 & 6.066 & 6.084 \\
\hline & $\mathrm{FM}$ & 45.27 & 5.66 & & & 5.51 & & & 677 & 6.401 & 6.391 \\
\hline Expt. & & 30.51 & 5.58 & 3.58 & 6.11 & & $0.010,0.203$ & $0.205,0.584$ & & & \\
\hline
\end{tabular}

this state are also close to the experimental values, indicating small influence of the noncollinear magnetic state of CrAs on its structural parameters. The MP-AFx state does not appear in Table I because this system, during structural relaxation, changes to the NA-AFx state.

Next, we consider bulk structures with epitaxial constraints. In these calculations, the in-plane lattice constants $a$ and $b$ are fixed by the epitaxial match to the GaAs substrate, while $c$ (along the surface normal) as well as the internal atomic positions are allowed to relax. The optimized values of $c$, called $c^{*}$, and the pertinent cohesive energy values are listed in Table I. In order to find out the appropriate geometrical patterns for matching the CrAs crystal structures to the $\operatorname{GaAs}(001)$ surface, we calculated the theoretical lattice parameter of ZB-GaAs within PBE-GGA and found a value
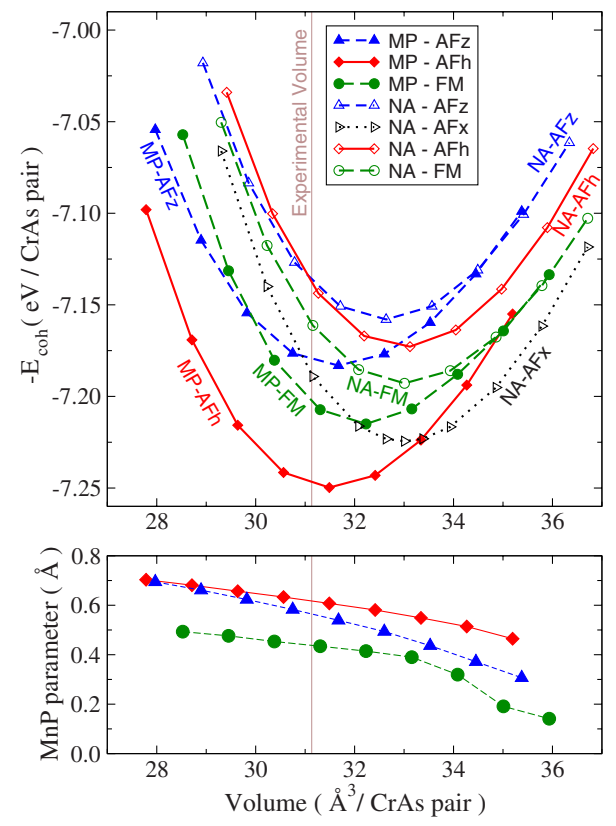

FIG. 3. (Color online) Top: energy-volume curves of MP- and NA-CrAs in different magnetic states. Bottom: variation in $\mathrm{MnP}$ parameter (vertical distance between $\mathrm{Cr} 2$ and $\mathrm{Cr} 1$ in MP-CrAs; see Fig. 1) as a function of volume in different magnetic states. of $5.74 \AA$. Hence, as shown in Fig. 4, the GaAs(001) surface lattice constant is $5.74 / \sqrt{2}=4.06 \AA$. The $\mathrm{GaAs}(001)$ surface unit-cell area (SUA), equal to $16.48 \AA^{2}$, is used as the unit of area throughout this paper. The calculated results (Table I) show that $\mathrm{ZB}-\mathrm{CrAs}$ has a lattice constant close to GaAs and thus can be matched directly to the GaAs(001) surface unit cell. Therefore, for the lattice-matched $\mathrm{ZB}-\mathrm{CrAs}$ and $\mathrm{ZB}$ $\mathrm{CrAs} / \mathrm{GaAs}(001)$ interface calculations a $(1 \times 1):(1 \times 1)$ pattern (see Fig. 4) was used. On the other hand, the calculated lattice parameters of MP- and NA-CrAs indicate that an appropriate and rather low strain pattern for matching these structures on $\mathrm{GaAs}(001)$ is to fit two $\mathrm{CrAs}(001)$ surface unit cells on three $\mathrm{GaAs}(001)$ surface unit cells (Fig. 4). This (2 $\times 1):(3 \times 1)$ pattern was used for lattice-matched MP- and NA-CrAs and MP-CrAs/GaAs(001) interface calculations. There is experimental evidence for the occurrence of this pattern in CrAs/GaAs(001) (Ref. 12) and MnAs/GaAs(001) (Ref. 22) heterostructures. We calculated and checked also $(1 \times 1):(1 \times 1)$ matched MP- and NA-CrAs structures on

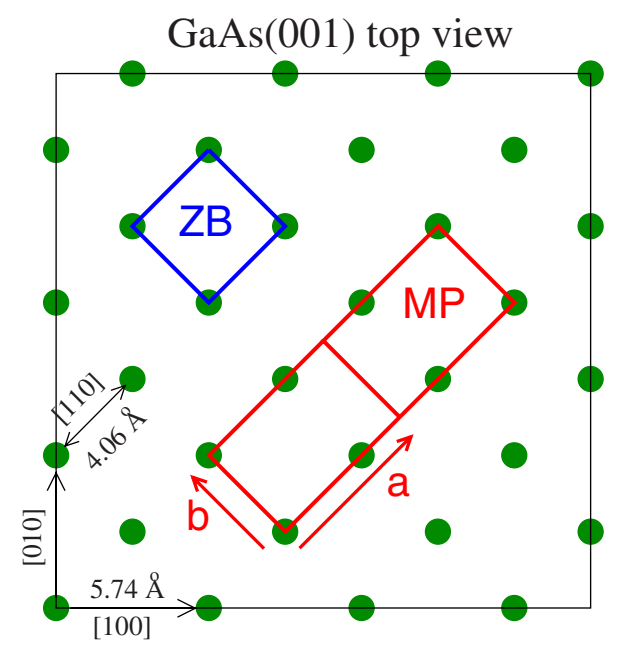

FIG. 4. (Color online) Schematic representation of the selected patterns for lattice-matched and interface calculations. For ZB-CrAs a $(1 \times 1):(1 \times 1)$ and for MP-CrAs and NA-CrAs a $(2 \times 1):(3$ $\times 1)$ pattern is selected. The matched MP (and NA) lattice parameters on the $\mathrm{GaAs}(001)$ surface are shown in the figure. 
$\operatorname{GaAs}(001)$ but found they are highly unstable.

Similar to the fully optimized MP-AFx structure, we observe that all MP lattice-matched structures relax to the corresponding NA structure. We found that this is a volume effect (Fig. 3, bottom): For all magnetic states of MP-CrAs, after increasing the volume, $\mathrm{Cr} 2$ moves down vertically toward the plane formed by the $\mathrm{Cr} 1$ atoms, and at some point the system undergoes a transition to the NA crystal structure. This structural modification of the lattice-matched systems may explain why Etgens et al. reported a modified orthorhombic structure in their molecular beam epitaxy-grown CrAs thin films on $\operatorname{GaAs}(001) .^{12}$

It is evidenced by the cohesive energies that in both the fully optimized and the lattice-matched structures the $\mathrm{ZB}$ phases are metastable and much higher in energy than the MP and NA lattices. The lattice-matched NA-FM state is found to be about $0.7 \mathrm{eV}$ more stable than the latticematched ZB-FM state. Therefore, the ZB structure can only originate from a nonequilibrium growth process. Moreover, we observe that AFh is the most stable magnetic state, even after matching to $\mathrm{GaAs}(001)$, and the FM state is $50 \mathrm{meV}$ higher in energy.

It is noteworthy that in the fully optimized MP lattices the FM state is found to be $35 \mathrm{meV}$ higher in energy than the stable AFh state while in the lattice-matched structures this differences rises to $50 \mathrm{meV}$. Thus we find that the strain induced by the substrate does not facilitate the formation of the FM state but stabilizes the AFh state even more. Before matching, the MP-AFh state is $20 \mathrm{meV}$ more stable than the second-most stable phase (NA-AFx) while after matching it becomes $43 \mathrm{meV}$ more stable than the next-stable phase (NA-AFz). Moreover, it is observed that strain is able to even change the order of stability of the magnetic states. As seen from Table I, in the fully optimized lattices, AFx and $\mathrm{AFz}$ are the second and the least stable magnetic states, respectively, while this order is reversed after matching.

The ZB phases of CrAs have considerably lower bulk moduli than the MP and NA phases. This finding is attributed to the lower coordination number of the atoms in the $\mathrm{ZB}$ structure. If $\mathrm{ZB}-\mathrm{CrAs}$ films could be stabilized, they will therefore be rather insensitive to strain.

\section{CrAs/GaAs(001) INTERFACE}

In this section, we study the properties of the epitaxial orthorhombic MP- and cubic ZB-CrAs thin films on the $\mathrm{GaAs}(001)$ substrate, taking the atomic structure of the interface into account. While the above results indicate that thick $\mathrm{ZB}$ films will be highly unstable, it is conceivable that the energetics of interface formation counterbalances this trend for films of only a few monolayers in thickness.

It was noted that biaxially strained MP-CrAs relaxes to the NA structure. Hence NA and MP-CrAs thin films relax to the same structure and need not to be considered separately. The MP-CrAs/GaAs(001) interfaces were simulated by using slab supercells consisting of $3 \mathrm{CrAs}+4 \mathrm{GaAs}$ layers. (Layers are counted according to the NiAs structure.) The ZB$\mathrm{CrAs} / \mathrm{GaAs}(001)$ interface was studied in slab supercells comprising $4 \mathrm{CrAs}+4 \mathrm{GaAs}$ layers. A vacuum thickness of

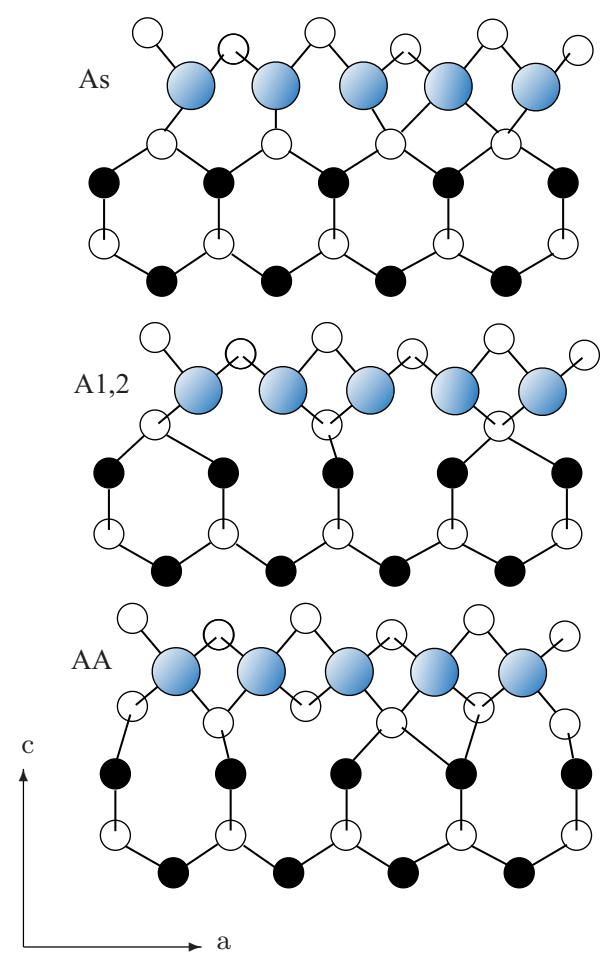

FIG. 5. (Color online) Schematic side views of the As, A1 and AA termination (top to bottom) of the MP-CrAs/GaAs(001) interface. The white, black, and grayish circles stand for As, $\mathrm{Ga}$, and $\mathrm{Cr}$ atoms, respectively.

about $10 \AA$ was used in all supercells to prevent interaction between adjacent slabs. The top (CrAs surface) and bottom (GaAs surface) layer of the slab consisted of As atoms. The arsenic dangling bonds could give rise to surface states that could affect interface properties. In order to saturate these broken bonds, the As surfaces of $\mathrm{GaAs}(001)$ and MP$\mathrm{CrAs}(001)$ were passivated by hydrogen atoms. In the case of the ZB-CrAs(001) surface, we tested two different ways to stabilize the surface, either passivation by hydrogen atoms, or dimerization of the surface As atoms, forming a $(2 \times 1)$ reconstruction of $\mathrm{ZB}-\mathrm{CrAs}(001)$. It is found that the reconstructed $\mathrm{ZB}$ thin film is more stable than the hydrogen passivated one.

Next, we discuss the atomic structure of the CrAs-GaAs interface. The experimental growth of this system is usually done under As-rich conditions which favors formation of an interface As layer. ${ }^{12,23}$ Moreover, our test calculations confirm the much higher stability of an As interface layer compared to a Cr-Ga-bonded interface. Adopting the As terminations along with the epitaxial morphology of the interface leads to only one possible structure for $\mathrm{ZB}-\mathrm{CrAs}$ thin films on $\mathrm{GaAs}(001)$ in which $\mathrm{Cr}$ atoms continue the $\mathrm{Ga}$ sublattice of the substrate. For the MP-CrAs/GaAs(001) interface, we considered four plausible interface structures (cf. Fig. 5) where the interface As layer results from (1) As termination of GaAs (denoted by suffix As), or (2) and (3) As terminations of CrAs by one of the two arsenic layers sandwiched between the $\mathrm{Cr}$ layers (denoted by suffix A1 or A2). In A1, the As atoms sit in hollow sites between $\mathrm{Ga}$ atoms while in A2 they sit in bridge sites of the interface Ga atoms. (4) As 


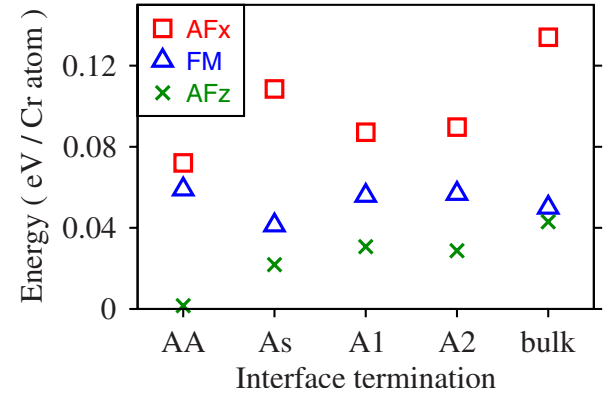

FIG. 6. (Color online) Total energies of different magnetic states for films with different interfaces (see text) of MP-CrAs/ $\mathrm{GaAs}(001)$, and for epitaxially strained bulk MP-CrAs. For each termination the energy of the AFh state has been selected as the reference energy.

termination by both As layers of CrAs (denoted by suffix AA). The concentration of the interface As atoms in A1, A2, As, and AA terminations are $1,2 / 3,2 / 3$, and $4 / 3$ atom/SUA, respectively.

In order to find out the stable structure and magnetic state of the interface, we applied the FM, AFh, $\mathrm{AFz}$, and $\mathrm{AFx}$ spin orders to all terminations of MP-CrAs/GaAs(001), and the FM state to the As termination of $\mathrm{ZB}-\mathrm{CrAs} / \mathrm{GaAs}(001)$. The total energies of the different magnetic states of the MPCrAs thin film are plotted in Fig. 6. It is observed that all interfaces prefer the AFh antiferromagnetic state, consistent with bulk CrAs. It is seen that the second-most stable state in all cases is $\mathrm{AFz}$, while $\mathrm{AFx}$ is the least stable state in all studied interface structures. We observe that in the AA termination, the $\mathrm{AFz}$ magnetic state has almost the same energy as the stable AFh state, while it has much higher total magnetic moment compared to other antiferromagnetic states (Table II). Hence it appears likely that a rather small external magnetic field can flip the spin of the interface $\mathrm{Cr}$ atoms and thus produce an enhanced magnetization in this system.

Our results about the energetic order of the magnetic states are in contrast to results by Arúajo et al. ${ }^{24}$ for freestanding CrAs films. On the basis of all-electron calculations for strained CrAs films with MP structure (but without substrate), these authors claimed that there is a transition from AF to FM ordering for a film thickness of $\sim 24 \AA$. Thus, CrAs films on a substrate behave clearly different from (hypothetical) free-standing films.

After finding the stable magnetic state of each interface, we compare the stability of different interface structures of
$\mathrm{CrAs} / \mathrm{GaAs}(001)$ in the framework of $a b$ initio atomistic thermodynamics. ${ }^{25}$ In this scheme, the film formation energy $\gamma$ per area (including both the interface and surface contribution, and strain energy contributions from the interior of the film) is used to address the thermodynamic stability of different thin film structures. The free energy $\gamma$ is defined as $\gamma=\left[E-\sum_{i} N_{i} \mu_{i}\left(T, p_{i}\right)\right] / A$, where $E$ is the DFT total energy ${ }^{26}$ of the slab supercell, after eliminating the energy contribution from the H-passivated GaAs substrate, $N_{i}$ and $\mu_{i}$ are the number and the chemical potential of the $i$ th element, $A$ is the interface area, and $i$ runs over $\mathrm{Cr}$, $\mathrm{As}$, and $\mathrm{H}$ atoms. In order to eliminate the energy contribution from the $\mathrm{H}$-passivated GaAs substrate, we calculated the energy of a GaAs slab that is terminated by $\mathrm{H}$ atoms on both sides, with fixed As-H bond length. This mirror symmetric slab had twice the number of layers that is used for GaAs substrate beneath the CrAs. Then one half of the total energy of this GaAs slab was subtracted from the CrAs/GaAs slab. For the remaining $\mathrm{H}$ atoms at the free As surface of CrAs, $\mu_{\mathrm{H}}$ was set to the binding energy per atom of the $\mathrm{H}_{2}$ molecule (4.475 eV). ${ }^{27}$ Assuming bulk CrAs as thermodynamic reservoir, the following equilibrium condition can be imposed: $\mu_{\mathrm{Cr}}+\mu_{\mathrm{As}}$ $=e_{b}$, where $e_{b}$ is the DFT total energy ${ }^{26}$ of bulk CrAs. Hence there is only one independent chemical potential in our system, which is selected to be $\mu_{\mathrm{As}}$. The thin-film free energies $\gamma$ calculated in this way are plotted as function of $\mu_{\mathrm{As}}$ in Fig. 7.

It is clearly visible that in the thermodynamically allowed region of the chemical potential ${ }^{28}$ the AA-terminated MP$\mathrm{CrAs} / \mathrm{GaAs}(001)$ film is most stable among the structures investigated, while the $\mathrm{ZB}-\mathrm{CrAs} / \mathrm{GaAs}(001)$ film has the lowest stability among the studied systems. The free energy difference of these two systems in the whole allowed region is more than $0.8 \mathrm{eV} / \mathrm{SUA}$, which is large compared to typical thermal energies during epitaxial growth. Therefore, as already expected from the bulk data, the growth of $\mathrm{ZB}-\mathrm{CrAs}$ films on $\operatorname{GaAs}(001)$ appears unlikely. However, the interface between CrAs and GaAs may not be atomically sharp. This seems plausible when considering that the GaAs(001) substrate used for growth is reconstructed, ${ }^{29}$ and shows atomic rows and trenches due to the reconstruction pattern. Then, a one or two-layer thin region of mixed composition $\mathrm{Ga}_{1-x} \mathrm{Cr}_{x} \mathrm{As}$ may form at the $\mathrm{CrAs} / \mathrm{GaAs}$ interface, in which $\mathrm{Cr}$ atoms are fourfold coordinated as in the $\mathrm{ZB}$ structure. We modeled such an intermediate region sandwiched between the GaAs substrate and the MP-CrAs film, choosing values of $x=1 / 3,2 / 3$, and 1 . The number of fourfold-coordinated $\mathrm{Cr}$

TABLE II. The total $\left(m_{t}\right)$ and absolute $\left(m_{a}\right)$ value of magnetization $\left(\mu_{B} / \mathrm{Cr}\right.$ atom) calculated for all four interfaces of MP-CrAs and for the As-interface of ZB-CrAs thin films on $\mathrm{GaAs}(001)$.

\begin{tabular}{|c|c|c|c|c|c|c|c|c|c|c|}
\hline & \multicolumn{8}{|c|}{ MP-CrAs } & \multicolumn{2}{|c|}{ ZB-CrAs } \\
\hline & \multicolumn{2}{|c|}{$\mathrm{AFh}$} & \multicolumn{2}{|c|}{$\mathrm{AFz}$} & \multicolumn{2}{|c|}{$\mathrm{AFx}$} & \multicolumn{2}{|c|}{ FM } & \multicolumn{2}{|c|}{ FM } \\
\hline & $m_{t}$ & $m_{a}$ & $m_{t}$ & $m_{a}$ & $m_{t}$ & $m_{a}$ & $m_{t}$ & $m_{a}$ & $m_{t}$ & $m_{a}$ \\
\hline A1 & -0.11 & 3.26 & 0.80 & 3.43 & -0.18 & 3.29 & 2.86 & 3.61 & & \\
\hline $\mathrm{A} 2$ & 0.11 & 3.45 & 0.86 & 3.58 & 0.11 & 3.50 & 2.81 & 3.68 & & \\
\hline As & 0.11 & 3.32 & 0.70 & 3.47 & 0.09 & 3.36 & 2.74 & 3.62 & 2.88 & 3.46 \\
\hline $\mathrm{AA}$ & 0.01 & 3.37 & 0.78 & 3.46 & 0.01 & 3.40 & 2.81 & 3.61 & & \\
\hline
\end{tabular}




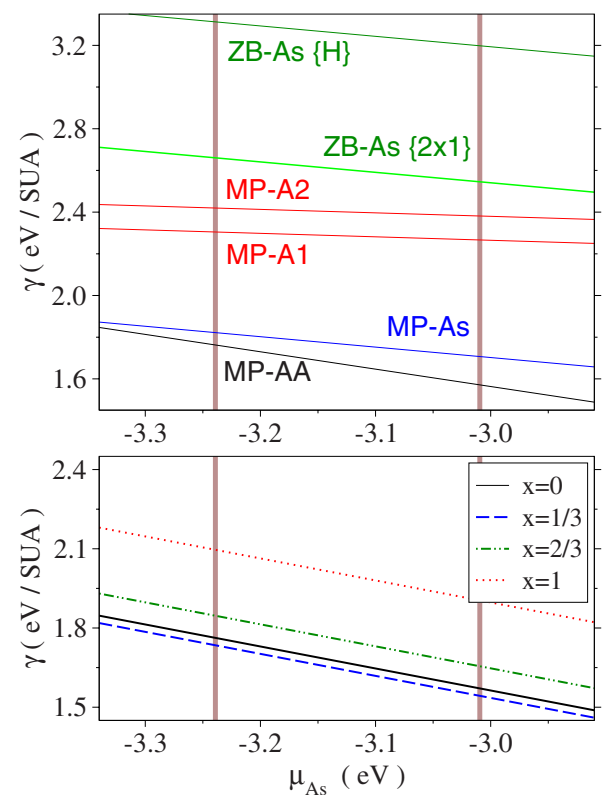

FIG. 7. (Color online) Calculated thin-film free energies of ZBCrAs and MP-CrAs thin films on GaAs(001) with atomically sharp interfaces (top panel) and with mixed interfaces of AA-terminated MP-CrAs (bottom panel). The thick vertical lines indicate the low and high limits of $\mu_{\mathrm{As}}$ and enclose the region accessible in thermodynamic equilibrium. The notations ZB-As $\{2 \times 1\}$ and ZB-As $\{\mathrm{H}\}$ indicate that the As surface of CrAs is stabilized by applying a $(2$ $\times 1$ ) reconstruction, or by hydrogen atoms, respectively.

atoms is proportional to $x$. All these modifications were applied to the AFh state of the AA-terminated MP-CrAs thin films. The magnetic moments of the fourfold-coordinated $\mathrm{Cr}$ atoms were all aligned in parallel, while the remaining $\mathrm{CrAs}$ layers were kept in the AFh state.

The interface free energies of these mixed interfaces are represented by the dashed lines in Fig. 7. Since all resulting free-energy curves are parallel to each other, it is sufficient to compare them at a fixed value of $\mu_{\mathrm{As}}$. In Fig. 8, the values of $\gamma$ are plotted as a function of the mixing parameter $x$, using a value of $\mu_{\mathrm{As}}=-3.13 \mathrm{eV}$, i.e., in the middle of the allowed region in Fig. 7. We observe that a mixing concentration of $x=1 / 3$ reduces the free energy and thus enhances the stability of the system. Inspection of the magnetic moments (Fig. 8 , right scale) shows that the uncompensated magnetic moments increase with increasing mixing concentration. The

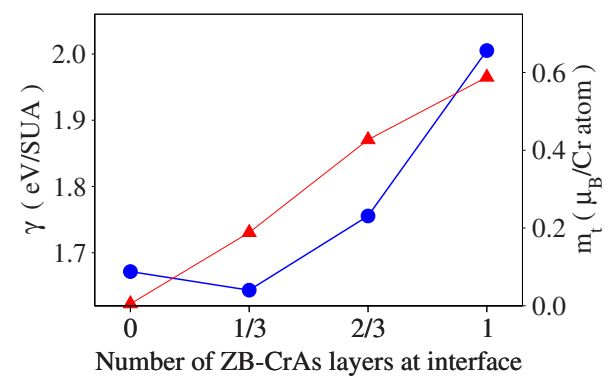

FIG. 8. (Color online) Calculated thin-film free energy $\gamma$ (circles) at $\mu_{\mathrm{As}}=-3.13 \mathrm{eV}$, and uncompensated magnetic moment $m_{t}$ (triangles) as a function of interface mixing concentration $x$.

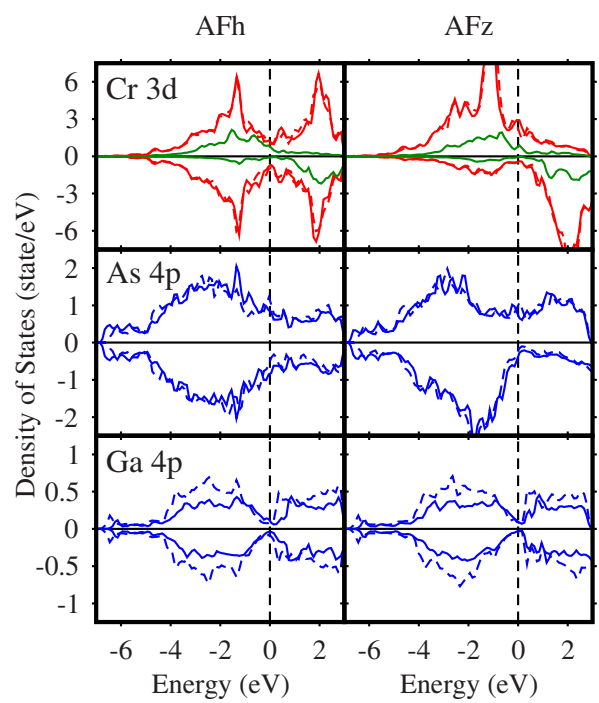

FIG. 9. (Color online) The spin-resolved partial density of states of the interface atoms in the most stable MP-AA (dashed line) and $x=1 / 3$ intermixed (solid lines) interface terminations with AFh (left column) and $\mathrm{AFz}$ (right column) spin orders. Positive and negative densities correspond to majority and minority states. The Fermi energies are set to zero.

mixed interface with $x=1 / 3$ which is found to be the most stable structure for MP-CrAs/GaAs(001) has a higher uncompensated moment compared to the film with the atomically sharp AA termination. Since in the AA-terminated MP$\mathrm{CrAs}$ thin film, the $\mathrm{AFz}$ state has a very close energy to the stable AFh state, we calculated the AFz state of the $x=1 / 3$ intermixed structure. It was observed that the $\mathrm{AFz}$ state of this stable mixed interface is about $4 \mathrm{meV} / \mathrm{Cr}$ atom $(16 \mathrm{meV} /$ SUA) less stable than the AFh state and its total uncompensated magnetic moment is about $0.93 \mu_{B} / \mathrm{Cr}$ atom.

The valence electronic structure of the most stable interfaces, the AFh and AFz states of the MP-AA and $x=1 / 3$ intermixed interfaces, are presented in Fig. 9. In order to avoid possible complications due to the presence of several atoms at these interfaces, we summed over the partial density of states (PDOS) of the same chemical species at the interface. For example, the plotted As $4 p$ PDOS in Fig. 9 is the sum of the $4 p$ PDOS of four As atoms at the interfaces. In the case of the mixed interface (solid lines), the PDOS for both inequivalent $\mathrm{Cr}$ atoms is plotted, the smaller one (green one) corresponding to the fourfold-coordinated $\mathrm{Cr}$ atom at the interface. The $4 s$ valence electrons of GaAs do not contribute to the DOS around the Fermi level and hence are omitted in this figure. We observe that the magnetic moments of the sixfold coordinated interface $\mathrm{Cr}$ atoms (large signals in the top left panel in Fig. 9, red lines) in the AFh state of both interfaces compensate each other and display a nonmagnetic DOS, while the in-plane ferromagnetic order in the $\mathrm{AFz}$ states gives rise to a considerable spin polarization at the Fermi level at the interface atoms. The fourfold-coordinated $\mathrm{Cr}$ atom in the mixed structure shows almost half-metallic behavior. A pseudogap is observed in the PDOS of the interface $\mathrm{Ga}$ atoms which is increased after mixing with the fourfold coordinated $\mathrm{Cr}$ atom. The increase in this pseudogap in the intermixed structure could explain the increased stability 

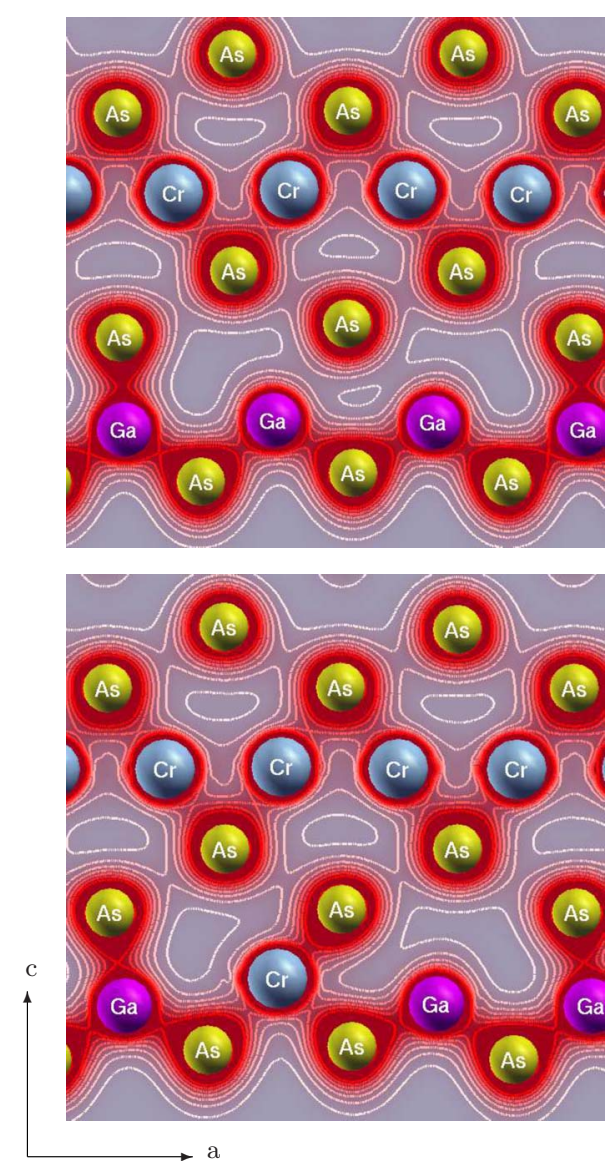

FIG. 10. (Color online) The total electron-density contour plots of the relaxed ideal AA (top) and $x=1 / 3$ intermixed (bottom) MP$\mathrm{CrAs} / \mathrm{GaAs}$ interfaces in the (010) plane. The white contour lines and the dark red areas around the atoms correspond to low and high electron charge-density regions, respectively. The width of the plots are somewhat larger than a unit cell, meaning that the right $\mathrm{Ga}$ atom is identical with the left $\mathrm{Ga}$ atom.

of this system compared with the ideal AA-terminated interface.

The electronic charge-density contour plots of the ideal MP-AA and $x=1 / 3$ intermixed interfaces in the (010) plane are plotted and compared in Fig. 10. As it is clearly visible and explained in the figure caption, one third of the interface $\mathrm{Ga}$ atoms in the $x=1 / 3$ intermixed interface are replaced with $\mathrm{Cr}$ atoms. We observe that the replaced $\mathrm{Cr}$ atom makes stronger bond with the neighboring As atom compared with the original gallium atom. This observation may explain the greater stability of the intermixed structure from the bonding point of view.

\section{MAGNETIC PROPERTIES}

The total and absolute magnetic moments of the ZB- and MP-CrAs thin films extracted from our calculations are given in Table II. The total $\left(m_{t}\right)$ and absolute $\left(m_{a}\right)$ magnetic moments are defined as $\int\left(\rho(\mathbf{r})_{\uparrow}-\rho(\mathbf{r})_{\downarrow}\right) d \mathbf{r}$ and $\int \mid \rho(\mathbf{r})_{\uparrow}$ $-\rho(\mathbf{r})_{\downarrow} \mid d \mathbf{r}$, respectively, where $\rho(\mathbf{r})$ is the electron density and the up/down arrows stand for the majority/minority spin states. In the ferromagnetic state the absolute and total magnetization should be close together, while for ideal antiferromagnetic order $m_{t}$ should vanish.

For all structures considered, the average absolute magnetic moments per $\mathrm{Cr}$ atom are in the range of $3.3 \mu_{B}$ to $3.5 \mu_{B}$. However, only in case of the ZB films a sizable total moment of $2.88 \mu_{B}$ in the magnetic ground state is found. In the films with MP-type structure, the energetically favorable antiparallel alignment of the $\mathrm{Cr}$ magnetic moments leads to a small total moment of about $0.1 \mu_{B}$ per $\mathrm{Cr}$ atom for $\mathrm{AFh}$ ordering, and about $0.8 \mu_{B}$ per $\mathrm{Cr}$ for $\mathrm{AFz}$ ordering. In the latter case, the compensation between the $\mathrm{Cr}$ moments of the three $\mathrm{Cr}$ layers of the film is not complete. The finite values of $m_{t}$ in antiferromagnetic MP-CrAs films quoted in Table II are attributed to this effect, termed uncompensated magnetic moment in the discussion that follows. Moreover, at mixed interfaces, the magnetic moments of fourfold-coordinated $\mathrm{Cr}$ atoms may couple ferromagnetically with one another, thus enhancing the uncompensated moment. The results presented in Fig. 8 indicate that at the most stable interface $(x=1 / 3)$ the uncompensated magnetic moment is about $0.2 \mu_{B}$ per $\mathrm{Cr}$ atom.

At first sight, it appears that only the ZB structure of the films is compatible with the experimentally observed ferromagnetic hysteresis of the samples. However, we believe that the true atomic structure of the films is not ideal ZB. First, the large energy difference found in our calculations between $\mathrm{ZB}$ and the MP ground state makes it unlikely that an ideal, defect-free ZB structure could be stabilized in thin-film growth (e.g., due to kinetic constraints). However, one has to keep in mind that the DFT-GGA functionals in some cases yield a too high energy difference (or even the wrong sign) when comparing systems with different atomic coordination and different magnetic ordering, e.g., $\mathrm{MnN}$ in rocksalt and $\mathrm{ZB}$ structure. ${ }^{30}$ This issue must be resolved by future work using improved DFT functionals.

More importantly, the experimental reports are not unanimous. While TEM images have been interpreted as indicating ZB structure in the films, ${ }^{9-11}$ a GIXD study concluded that the films have a strained orthorhombic structure. ${ }^{12,23}$ In addition, we argue that ideal $\mathrm{ZB}$ CrAs, if at all present in the films, is expected to show a hysteretic signal different from what is actually observed in the experiments: The common and most important feature of all experiments on CrAs thin films on $\mathrm{GaAs}(001)$ is the observation of a very narrow, S-shaped hysteresis loop with high saturation magnetization but low remanent magnetization. Inspection of the reported measured data shows that the remanent magnetization is always less than $10 \%$ of the saturation magnetization. Such a behavior could be rationalized in three ways: either the external magnetic field is applied in the direction of the hard axis or the system is a weak ferromagnet, or ferromagnetic order exists only in a small part of the system. Since the thin films were magnetized in-plane ${ }^{10}$ and one would not expect a strong intrinsic magnetocrystalline anisotropy for a $3 d$ compound such as CrAs, the first explanation, magnetization along a hard axis, is unlikely. Moreover, from a theoretical analysis, one would expect that CrAs in the $\mathrm{ZB}$ structures is not a weak ferromagnet. This becomes clear from the strength of the exchange interaction in ZB-CrAs. In the sim- 
plified picture of a Heisenberg model with nearest-neighbor interaction, $H=J\left(M_{1} \cdot M_{2}\right)$, where $M_{1}$ and $M_{2}$ are the magnetic moments of neighboring $\mathrm{Cr}$ atoms, the energy difference between FM and AF states is a measure of the exchange interaction coefficient $J=\left(E_{\mathrm{AF}}-E_{\mathrm{FM}}\right) /|M|^{2}$. Using the calculated energies in Table I for lattice-matched ZB-CrAs, $J$ is estimated to be $15 \mathrm{meV}$, in good agreement with previous calculations. ${ }^{31}$ This nearest-neighbor exchange interaction in $\mathrm{ZB}-\mathrm{CrAs}$ is one order of magnitude larger than for some strong ferromagnets such as $\mathrm{Co}_{2} \mathrm{MnSi}$ Heusler alloy, ${ }^{32}$ although the range of exchange interaction is considerably shorter in $\mathrm{ZB}-\mathrm{CrAs} .{ }^{31}$ Due to this strong exchange interaction, a high Curie temperature of about $1000 \mathrm{~K}$ for $\mathrm{ZB}-\mathrm{CrAs}$ is predicted by first-principles calculations, ${ }^{33}$ which has not been confirmed by experiments up to now.

Excluding the first two possible explanations for the shape of the hysteresis loop, we favor the third of the abovementioned possibilities: We attribute the observed narrow hysteresis in thin CrAs films to ferromagnetic order in small, weakly coupled domains, which would lead to an almost superparamagnetic behavior of the samples, as evident from the measured hysteresis.

From these considerations, we arrive at some suggestions for directions of future work. We believe that incomplete compensation of magnetic moments plays an important role in the magnetic phenomena in thin CrAs films and requires further investigations. The total magnetic moments quoted in Table II likely underestimate the magnetization to be expected in real samples. First, the formation of spin spiral structures known for bulk CrAs has been neglected in the present study. Truncation of a spin spiral structure in ultrathin films is an obvious source of noncompensating magnetic moments. Second, the structural complexity of real samples, e.g., due to domain boundaries and interface reconstruction, could further enhance the amount of uncompensated magnetic moments. We note that the GaAs(001) substrate surface is reconstructed. The trench-and-hill pattern of the surface reconstruction could lead to an enhanced intermixing near the $\mathrm{CrAs} / \mathrm{GaAs}$ interface. As we showed by the results in Fig. 8, intermixing gives rise to larger total magnetic moments. The possible role of the interface for the magnetic properties is in agreement with the observation of Etgens et $a l .{ }^{12}$ that the average magnetic moment per $\mathrm{Cr}$ atom tends to zero for thicker CrAs films, i.e., the "ferromagnetism" occurs only in the first few layers of CrAs thin films. Likewise, Mosca et al. ${ }^{23}$ speculated that the predominant contribution to the magnetism of the CrAs thin films comes from a very thin interface layer. A theoretical description of the interface reconstruction and the spin spiral structure would require large unit cells, leading to a huge computational demand, and lies therefore outside the scope of this work.

Finally, we point out an analogy between the magnetism in CrAs thin films and observations in exchange-bias systems ${ }^{34}$ or composite materials made of antiferromagnetic nanoparticles. Already in 1961, Neel predicted that the lack of compensation in small AF nanoparticles may give rise to a weak ferromagnetic behavior. ${ }^{35}$ Following his prediction, small magnetic hysteresis loops were observed in some AF nanoparticular systems. ${ }^{36,37}$ Surface enhancement of atomic magnetic moments produces a net magnetization and consequently a weak ferromagnetic hysteresis in the antiferromagnetic nanoparticles. Mørup, in 1985, suggested a theoretical model to justify that even a small unbalance of magnetic moments of about $0.5 \%$ may lead to a measurable ferromagnetic hysteresis in AF nanoparticles. ${ }^{38}$ In the present system, three monolayers of MP-CrAs on $\mathrm{GaAs}(001)$, the unbalance is much larger, e.g., $\sim 6 \%$ in the AFh state with mixed interface. Moreover, it is remarkable that the thermoremanent behavior found in some CrAs thin films ${ }^{23}$ also shows similarities to observations in nanoparticle systems: field-cooled samples display an increase in magnetization below room temperature while samples cooled in the absence of a magnetic field show only little temperature dependence of their magnetization. This observation could indicate the existence of weakly coupled magnetic domains.

\section{SUMMARY}

First-principles calculations using density-functional theory have been performed to study the structural and magnetic properties of bulk CrAs, as well as CrAs thin films on $\mathrm{GaAs}(001)$. In the bulk calculations it was found that the bulk ground state, an orthorhombic MnP-type structure with antiferromagnetic spin order, persists even if epitaxial constraints are taken into account. In strained epitaxial films, an analogous structure (but without the rumpling in the $\mathrm{Cr}$ layers) is found to be most stable. It shows layered antiferromagnetism, but with some net magnetization due to incomplete compensation of the $\mathrm{Cr}$ magnetic moments. The ferromagnetic zinc-blende structure is considerably higher in energy, and hence is unlikely to be stabilized under epitaxial conditions. We conclude that none of the calculated homogeneous crystalline films matches the experimentally observed ferromagnetic hysteresis. The narrowness of the measured hysteresis loop is suggestive of inhomogeneous samples with uncompensated magnetic moments at the $\mathrm{Cr}$ atoms near grain boundaries and near the interface to GaAs. For spintronics applications, these results imply that CrAs is probably not a good material for fabrication of macroscopically thick ferromagnetic layers that could serve as spin injectors. However, since the above conclusions rest on relatively small energy differences, the results are material specific, and other $\mathrm{Cr}$ pnictides on semiconductor substrates might perform better in this respect.

\section{ACKNOWLEDGMENTS}

S.J.H. was supported by the Alexander-von-Humboldt Stiftung. We acknowledge financial support by Deutsche Forschungsgemeinschaft within SFB491 "Magnetic heterostructures: Spin structure and spin transport." 
${ }^{1}$ M. Zwierzycki, K. Xia, P. J. Kelly, G. E. W. Bauer, and I. Turek, Phys. Rev. B 67, 092401 (2003).

${ }^{2}$ V. P. LaBella, D. W. Bullock, Z. Ding, C. Emery, A. Venkatesan, W. F. Oliver, G. J. Salamo, P. M. Thibado, and M. Mortazavi, Science 292, 1518 (2001).

${ }^{3}$ G. Schmidt, D. Ferrand, L. W. Molenkamp, A. T. Filip, and B. J. van Wees, Phys. Rev. B 62, R4790 (2000).

${ }^{4}$ H. Akinaga, T. Manago, and M. Shirai, Jpn. J. Appl. Phys., Part 2 39, L1118 (2000).

${ }^{5}$ P. Mavropoulos, M. Ležaić, and S. Blügel, Phys. Rev. B 72, 174428 (2005).

${ }^{6}$ M. Mizuguchi, H. Akinaga, T. Manago, K. Ono, M. Oshima, M. Shirai, M. Yuri, H. J. Lin, H. H. Hsieh, and C. T. Chen, J. Appl. Phys. 91, 7917 (2002).

${ }^{7}$ M. Mizuguchi, H. Akinaga, T. Manago, K. Ono, M. Oshima, and M. Shirai, J. Magn. Magn. Mater. 239, 269 (2002).

${ }^{8}$ H. Ofuchi, M. Mizuguchi, K. Ono, M. Oshima, H. Akinaga, and T. Manago, Nucl. Instrum. Methods Phys. Res. B 199, 227 (2003).

${ }^{9}$ H. Akinaga and M. Mizuguchi, J. Phys.: Condens. Matter 16, S5549 (2004).

${ }^{10}$ J. F. Bi, J. H. Zhao, J. J. Deng, Y. H. Zheng, S. S. Li, X. G. Wu, and Q. J. Jia, Appl. Phys. Lett. 88, 142509 (2006).

${ }^{11}$ J.-F. Bi, J.-H. Zhao, J.-J. Deng, Y.-H. Zheng, W.-Z. Wang, J. Lu, Y. Ji, and S.-S. Li, Chin. Phys. 16, 3868 (2007).

${ }^{12}$ V. H. Etgens, P. C. de Camargo, M. Eddrief, R. Mattana, J. M. George, and Y. Garreau, Phys. Rev. Lett. 92, 167205 (2004).

${ }^{13}$ K. Selte, A. Kjekshus, W. E. Jamison, A. F. Andresen, and J. E. Engebretsen, Acta Chem. Scand. (1947-1999) 25, 1703 (1971).

${ }^{14}$ W.-H. Xie, Y.-Q. Xu, B.-G. Liu, and D. G. Pettifor, Phys. Rev. Lett. 91, 037204 (2003).

${ }^{15}$ Y.-J. Zhao and A. Zunger, Phys. Rev. B 71, 132403 (2005).

${ }^{16}$ K. Nagao, M. Shirai, and Y. Miura, J. Appl. Phys. 95, 6518 (2004).

${ }^{17}$ P. Giannozzi et al., J. Phys.: Condens. Matter 21, 395502 (2009).

${ }^{18}$ QUANTUM-ESPRESSO is a community project for high-quality quantum simulation software, based on DFT and coordinated by Paolo Giannozzi. See http://www.quantum-espresso.org and http://www.pwscf.org

${ }^{19} \mathrm{We}$ used the pseudopotentials Cr.pbe-sp-van.UPF, As.pbe-nvan.UPF, and Ga.pbe-nsp-van.UPF from http://www.quantumespresso.org distribution.

${ }^{20}$ P. Blaha, K. Schwarz, G. Madsen, D. Kvasnika, and J. Luitz,
WIEN2k, An Augmented Plane Wave Plus Local Orbitals Program for Calculating Crystal Properties (Technische Universitat Wien, Vienna, 2001).

${ }^{21}$ J. P. Perdew, K. Burke, and M. Ernzerhof, Phys. Rev. Lett. 77, 3865 (1996).

${ }^{22}$ A. K. Das, C. Pampuch, A. Ney, T. Hesjedal, L. Däweritz, R. Koch, and K. H. Ploog, Phys. Rev. Lett. 91, 087203 (2003).

${ }^{23}$ D. H. Mosca, P. C. de Camargo, J. L. Guimarães, W. H. Schreiner, A. J. A. de Oliveira, P. E. N. Souza, M. Eddrief, and V. H. Etgens, J. Phys.: Condens. Matter 17, 6805 (2005).

${ }^{24}$ A. A. Araújo, B. Laks, and P. C. de Camargo, Phys. Rev. B 74, 172411 (2006).

${ }^{25}$ K. Reuter, C. Stampfl, and M. Scheffler, in Handbook of Materials Modeling, edited by S. Yip (Springer, Berlin, 2005), pp. 149-194, and references therein.

${ }^{26}$ Here the Gibbs free energy may be approximated by the DFT total energy because at sufficiently low temperatures often the vibrational contributions to the Gibbs free energy have negligible impact on energy differences of different phases (see Ref. 25).

${ }^{27}$ W. Martienssen and H. Warlimont, Springer Handbook of Condensed Matter and Materials Data (Springer-Verlag, Berlin, 2005).

${ }^{28}$ The value of the As chemical potential is practically limited by the As-rich and Cr-rich boundaries which correspond to the formation of bulk As and $\mathrm{Cr}$ structures. In order to determine the boundaries we calculated the ground-state energy of optimized bulk $\mathrm{Cr}$ and As structures. For a detailed discussion, see Ref. 25.

${ }^{29}$ S.-H. Lee, W. Moritz, and M. Scheffler, Phys. Rev. Lett. 85, 3890 (2000).

${ }^{30}$ J. A. Chan, J. Z. Liu, H. Raebiger, S. Lany, and A. Zunger, Phys. Rev. B 78, 184109 (2008).

${ }^{31}$ E. Şaşığlu, I. Galanakis, L. M. Sandratskii, and P. Bruno, J. Phys.: Condens. Matter 17, 3915 (2005).

${ }^{32}$ E. Şaşıŏlu, L. M. Sandratskii, P. Bruno, and I. Galanakis, Phys. Rev. B 72, 184415 (2005).

${ }^{33}$ J. Kübler, Phys. Rev. B 67, 220403(R) (2003).

${ }^{34}$ K. Takano, R. H. Kodama, A. E. Berkowitz, W. Cao, and G. Thomas, Phys. Rev. Lett. 79, 1130 (1997).

${ }^{35}$ L. Neel, Compt. Rend. 252, 4075 (1961).

${ }^{36}$ S. Makhlouf, F. Parker, F. Spada, and A. Berkowitz, J. Appl. Phys. 81, 5561 (1997).

${ }^{37}$ L. Zhang, D. Xue, and C. Gao, J. Magn. Magn. Mater. 267, 111 (2003).

${ }^{38}$ S. Mørup, Surf. Sci. 156, 888 (1985). 